\title{
Long non-coding RNA 00858 knockdown alleviates bladder cancer via regulation of the miR-3064-5p/CTGF axis
}

\author{
JI HUANG $^{1,2^{*}}$, QIU-MING HE ${ }^{2 *}$, QI WU ${ }^{3}$, WEI-MIN ZHOU ${ }^{2}$, CHAO HAO $^{2}$, GONG-XIAN WANG $^{1}$ and XIN-HUA TU ${ }^{2}$ \\ ${ }^{1}$ Department of Urology, The First Affiliated Hospital of Nanchang University, Nanchang, Jiangxi 330006; \\ Departments of ${ }^{2}$ Urology and ${ }^{3}$ Abdominal Surgery, Jiangxi Cancer Hospital, Nanchang, Jiangxi 330029, P.R. China
}

Received February 19, 2020; Accepted April 2, 2021

DOI: $10.3892 /$ or.2021.8115

\begin{abstract}
The long non-coding RNA 00858 (LINC00858) has been reported to be an oncogene for various cancer diseases, including osteosarcoma and colorectal cancer. However, the expression pattern and function of LINC00858 in bladder cancer remain largely unknown. The expression level of LINC00858 was measured in tumor tissues and cell lines by RT-qPCR. The role of LINC00858 in bladder cancer cells were studied by gain- and loss-of-function strategies in vitro. Cell proliferation, migration and invasion were assessed by CCK-8, colony formation, wound healing and Transwell chamber assays. At the molecular level, dual luciferase reporter and RNA RIP assays were performed to identify the interaction among LINC00858, microRNA (miR)-3064-5p and cellular communication network factor 2 (CTGF). The results revealed that the expression level of LINC00858 was upregulated in bladder cancer tissues and cell lines including T24, J82 and 5637. Moreover, knockdown of LINC00858 suppressed cell proliferation, migration and invasion in vitro. Mechanistically, LINC00858 functioned as a competitive RNA to increase the expression level of oncogene CTGF by sequestering miR-3064-5p. In conclusion, LINC00858 knockdown inhibited the proliferation, migration and invasion of bladder cancer cells via regulation of the miR-3064-5p/CTGF axis.
\end{abstract}

Correspondence to: Dr Gong-Xian Wang, Department of Urology, The First Affiliated Hospital of Nanchang University, 17 Yongzheng Street, Nanchang, Jiangxi 330006, P.R. China

E-mail: gongxianwang118@163.com

Dr Xin-Hua Tu, Department of Urology, Jiangxi Cancer Hospital, 519 East Beijing Road, Nanchang, Jiangxi 330029, P.R. China

E-mail: zhou629812@163.com

${ }^{*}$ Contributed equally

Key words: bladder cancer, long non-coding RNA 00858, proliferation, migration, invasion

\section{Introduction}

Bladder cancer is theninth most frequently diagnosed cancer globally, with an estimated 429,000 new cases and 165,000 cancer-related deaths worldwide in 2012 (1,2). Notably, a significant difference in the incidence rate between sexes exists; men always suffer from a higher incidence than women due to their addiction with smoking $(3,4)$. Most bladder cancers (75-80\%) that do not involve the muscular wall of the bladder usually obtain favorable clinical results through endoscopic treatment (4). In addition, radical cystectomy combined with abdominal lymph node dissection is the gold standard for the treatment of invasive bladder cancer. However, due to the difficulty of this surgery and the large number of postoperative complications, it is not suitable for all patients (5). Therefore, it is necessary to study the diagnosis and pathogenesis of bladder cancer. Exploring potential targets for bladder cancer treatment is urgently needed to further improve the therapeutic effects on bladder cancer.

In recent years, growing evidence has revealed that long non-coding RNAs act as biomarkers for diagnosis and prognosis of cancer patients, thus providing new therapeutic targets for cancer treatment (6-8). Numerous lncRNAs are aberrantly expressed in various tumors, and a number of lncRNAs, such as MALAT1 in lung cancer, are stable in body fluids and could be detected in the plasma and urine of patients with cancer (9-11). Increasing evidence has demonstrated that lncRNAs participate in bladder cancer formation, progression, and metastasis $(12,13)$. Wei et al have reported thatlncRNA MBNL1-AS1 suppressed cell proliferation and enhanced cell apoptosis via targeting of the microRNA (miR)-135a-5p/PHLPP2/FOXO1 axis in bladder cancer (14). Among these lncRNAs, LINC00858, located in 10q23.1, has been reported in three types of tumors, including lung cancer, osteosarcoma and colorectal cancer (15). In these malignancies, LINC00858 has been revealed to induce tumor progression and metastasis. However, the role of LINC00858 in the regulation of bladder cancer remains unknown.

In the present study, the expression level of LINC00858 was detectedin bladder cancer tissues and cells. Then, LINC00858 was knocked down by RNAi technology, and abilities including cell proliferation, migration and invasion were investigated. Futhermore, the mechanism underlying LINC00858 in bladder cancer was studied. 


\section{Materials and methods}

Human samples and ethical approval. Tumor tissues and paired adjacent normal tissues were obtained from 60 bladder cancer patients ( 28 females and 32 males) from June 2011 to July 2019 who underwent surgery at Jiangxi Cancer Hospital (Nanchang, China). The inclusion criteria was as follows: Age, from 28 to 61 years; histologically confirmed non-muscular invasive urothelial carcinoma of the bladder. The exclusion criteria was as follows: Patients with other concurrent uropoiesis reproductive system tumors; patients with severe cardiovascular and cerebrovascular disease such as heart failure; known immunodeficiency; various mental disorders. The present study was approved by the Ethics Committee of Jiangxi Cancer Hospital (approval no. 2019014). Written informed consent was obtained from all participants. All the tissue samples were collected and frozen in liquid nitrogen, and then stored at $-80^{\circ} \mathrm{C}$ for further use. The clinicopathological data of the patients are presented in Table SI.

Cell culture. Three human bladder cancer cell lines,T24, J82 and 5637 and a normal human bladder epithelial cell line SV-HUC-1, were purchased from the American Type Culture Collection (ATCC). All cell lines were cultured in RPMI-1640 medium supplemented with $10 \%$ fetal bovine serum (both from Invitrogen; Thermo Fisher Scientific, Inc.) at $37^{\circ} \mathrm{C}$ with $5 \% \mathrm{CO}_{2}$.

Cell transfection. The bladder cancer cell lines 5637 and T24 were respectively transfected with $0.2 \mu \mathrm{g}$ LINC00858 overexpression vector, 50 pmol LINC00858 short hairpin (sh)RNA, 50 pmol negative control (NC) shRNA, $50 \mathrm{pmol}$ CTGF shRNA, 50 pmol NC mimic (a non-targeting miR-scramble), 50 pmol miR-3064-5p mimic, 50 pmol miR-3064-5p inhibitor, $50 \mathrm{pmol}$ miR-NC inhibitor and $0.2 \mu \mathrm{g}$ empty vector was used as a control for the overexpression experiments (all from Shanghai GenePharma Co., Ltd. The sequences were: sh-LINC00858 (5'-GCGACATTAATG GGAATGA-3'); sh-CTGF (forward, 5'-CACCGCACCAGA ATGTATATTAATTCAAGAGATTAATATACATTCTGG TGCTTTTTTG-3' and reverse, 5'-GATCCAAAAAAGCAC CAGAATGTATATTAATCTCTTGAATTAATATACATT CTGGTGC-3'); sh-RNA NC (5'-GTTCTCCGAACGTGT CACGT-3'); miR-3064-5p mimic (5'-TTACTGGCTGTT GTGGTGTGC-3'); NC mimic (forward, 5'-UUCUCCGAA CGUGUCACGUTT-3' and reverse, 5'-ACGUGACACGUU CGGAGAATT-3'); miR-3064-5p inhibitor (5'-UUGCAC ACCACAACAGCCAGA-3'); and miR-NC inhibitor (5'-CAG UACUUUUGUGUAGUACAA-3'). Lipofectamine 2000 (Invitrogen; Thermo Fisher Scientific, Inc.) was used for transfection according to the manufacturer's protocols. After transfection for $6 \mathrm{~h}$ at $37^{\circ} \mathrm{C}$, fresh culture medium was added and cells were cultured for $48 \mathrm{~h}$ before futher experiments. Real-time PCR and green fluorescence microscopy were used to assess the transfection efficiency.

Bioinformatics analysis. To predict the interaction site of miR-3064-5p with LINC00858, starBase v2.0 (http://starbase. sysu.edu.cn/) (16) was employed according to the online instructions. In addition, the binding site between miR-3064-5p and CTGF was predicted by searching starBase v2.0 online.

CCK- 8 and colony formation assays. Cell proliferation was detected by CCK- 8 and colony formation assays. The CCK-8 assay was performed according to the manufacturer's instructions (Beyotime Biotechnology). In brief, 5,000 cells were seeded into a 96-well plate and cultured for 24, 48 and $72 \mathrm{~h}$. Then $5 \mu \mathrm{l}$ of CCK-8 reagent was added into each well and cultured for another $1 \mathrm{~h}$ at $37^{\circ} \mathrm{C}$ before detection on a microplate reader. The optical density (OD) values were then measured at $450 \mathrm{~nm}$. For the colony formation assay, 500 cells were seeded into a 6 -well plate and cultured for $\sim 14$ days, and then the colonies ( $>5$ cells per colony) were stained with $0.5 \%$ crystal violet at room temperature for $10 \mathrm{~min}$ and counted with a stereomicroscope (magnification, $x 40$; Leica MZ8; Leica Microsystems GmbH).

Wound healing assay. Approximately 20,000 cells were seeded into a 24 -well plate and cultured at $37^{\circ} \mathrm{C}$ for $24 \mathrm{~h}$. The scratch wounds were created in 5637 and T24 cells by $10-\mu 1$ pipette tips. The cells were washed 3 times with PBS, to remove the marked cells, and serum-free medium was added for culture. Representative images were captured after $48 \mathrm{~h}$ using a microscope (magnification, x200; Leica MZ8; Leica Microsystems $\mathrm{GmbH}$ ).

Transwell chamber assay. Approximately 20,000 cells in serum-free medium were seeded in the upper Transwell chamber ( $8 \mu \mathrm{m}$; Cell Biolabs, Inc.) with a Matrigel-coated membrane (pre-coated at $4^{\circ} \mathrm{C}$ for $12 \mathrm{~h}$ ) for invasion assays or in chambers not coated with Matrigel for migration assays, while the lower chamber was filled with complete medium as a chemoattractant. After $24 \mathrm{~h}$ at $37^{\circ} \mathrm{C}$, the cells on the upper part of the filters were removed with a cotton swab. The migrated and invasive cells were counted in 5 different fields (magnification, x200) under a light microscope (Nikon Corporation) after staining with $0.5 \%$ crystal violet at $37^{\circ} \mathrm{C}$ for $15 \mathrm{~min}$.

Dual luciferase reporter assay. To assess the relationship between CTGF and miR-3064-5p (or LINC00585), the online tool LncTar (http://www.cuilab.cn/lnctar) (17) was used to predict the binding site between CTGF 3'UTR (or LINC00585) and miR-3064-5p. Then, a dual-luciferase reporter assay was carried out. First, CTGF 3'-UTR was cloned into the downstream of the psiCheck 2 vector (Promega Corporation) to generate the wild-type CTGF 3'-UTR luciferase reporter vector; a mutant CTGF 3'-UTR luciferase reporter vector was generated by mutating the predicted miR-3064-5p binding site within the CTGF 3'-UTR. These two reporter vectors were cotransfected by Lipofectamine 2000 (Invitrogen; Thermo Fisher Scientific, Inc.) with 50 pmol of miR-3064-5p mimics (Shanghai GenePharma Co., Ltd.) into 5637 and T2 4 cells. Then, $48 \mathrm{~h}$ later, the cells were harvested and underwent a dual-luciferase reporter assay (Promega Corporation) to evaluate firefly and Renilla the luciferase activities. Renilla luciferase activity served as a normalization control.

RNA immunoprecipitation (RIP) assay. The Immunoprecipitation Kit (cat. no. 17-10085; EMD Millipore) was used for an RNA 
immunoprecipitation (RIP) assay according to the manufacturer's instructions. Briefly, the lysed cells $\left(5 \times 10^{6}\right)$ were incubated in $100 \mu \mathrm{l}$ of RIP buffer solution (cat. no. KT102-01; GZSCBio Co. Ltd.), and the magnetic beads (included in the kit) were labeled with anti-Ago 2 or IgG antibodies (included in the kit) at $4^{\circ} \mathrm{C}$ for $2 \mathrm{~h}$. The abundance of LINC00858, miR-3064-5p, and CTGF were verified by RT-qPCR.

Determination of reactive oxygen species (ROS). Approximately $1 \times 10^{5}$ cells were seeded into a 6 -well plate and cultured at $37^{\circ} \mathrm{C}$ for 24 h. Then CellROX ${ }^{\mathrm{TM}}$ Green reagent (ThermoFisher Scientific, Inc.) was added at a 1/500 volume of medium and cultured for $1 \mathrm{~h}$ at room temperature. After being washed with PBS buffer (Gibco; Thermo Fisher Scientific, Inc.), cells were monitored under a fluorescence microscope (Olympus BX43;Olympus Corporation) and the ROS content was calculated according to the control.

Reverse transcription-quantitative polymerase chain reaction $(R T-q P C R)$. Total RNA from tissues or target cells was extracted using TRIzol reagent (Invitrogen; Thermo Fisher Scientific, Inc.), following the manufacturer's procedures. A NanoDrop-1000 (Thermo Fisher Scientific, Inc.) was used to determine concentrations and for quality control purposes. Complementary DNA was synthesized from extracted RNA $(5 \mu \mathrm{g})$ using the Prime Script ${ }^{\circledR}$ RT Reagent kit with gDNA Eraser (Invitrogen; Thermo Fisher Scientific, Inc.) in accordance with the recommended protocol (samples and reagent mix were incubated at $37^{\circ} \mathrm{C}$ for $15 \mathrm{~min}$ and $85^{\circ} \mathrm{C}$ for $5 \mathrm{sec}$, and finally stored at $4^{\circ} \mathrm{C}$ ). qPCR was performed using $\mathrm{SYBR}^{\circledR}$ Premix Ex Taq ${ }^{\mathrm{TM}}$ II (Invitrogen; Thermo Fisher Scientific, Inc.). An ABI 7500 real-time PCR detection system (Applied Biosystems; Thermo Fisher Scientific, Inc.) was used for detection. The qPCR thermocycling conditions were as follows: The initial denaturation was first performed at $95^{\circ} \mathrm{C}$ for 2 min followed by denaturation at $95^{\circ} \mathrm{C}$ for $15 \mathrm{sec}$ and annealing and extension at $60^{\circ} \mathrm{C}$ for $30 \mathrm{sec}$. Then, denaturation, annealing and extension were repeated for 40 cycles. After amplification, data were collected and processed by the comparative cycle threshold method. U6 and GAPDH expression levels were used as internal references for miRNA and mRNA expression detection, respectively. Finally, the data were processed using the $2^{-\Delta \Delta C q}$ relative expression method (18). The PCR amplification products were quantified by the FastStart Universal SYBR Green Master (Roche Diagnostics) and normalized to GAPDH or U6.

Western blotting. Total protein was extracted from cultured cells using RIPA buffer (Beyotime Biotechnology) containing protease and phosphotase inhibitorcocktails. A BCA protein assay kit (Beyotime Biotechnology) was used to determine protein concentrations according to the manufacturer's instructions. Approximately $10 \mu \mathrm{g}$ of proteins were separated by 12\% SDS-PAGE and transferred to PVDF (EMD Millipore) membranes. The membranes were blocked with $5 \%$ non-fat milk at room temperature for 1 hand then incubated with primary antibodies overnight at $4^{\circ} \mathrm{C}$, and subsequently incubated with HRP-conjugated secondary antibodies (cat. no. A0216; dilution 1:5,000; Beyotime Biotechnology) at room temperature for $1 \mathrm{~h}$. Proteins were visualized using
ECL Western blotting detection reagent (EMD Millipore). Immunoreactive bands were quantified using Image J (1.8.0; $\mathrm{NIH})$. The following primary antibodies were used: Anti-Cox-2 (product code ab179800; 1:1,000), anti-MMP2 (product code ab92536; 1:2,000), anti-MMP9 (product code ab76003; $1: 2,000)$, anti-CTGF (product code ab209780; 1:1,000) and $\beta$-actin (product code ab8226; 1:1,000) (all from Abcam).

Statistical analysis. All data are presented as the mean \pm standard deviation and all experiments were repeated three times. Statistical significance between normal and tumor tissues was analyzed using a paired t-test, and other comparisons between two groups were analyzed by unpaired t-test. One-way ANOVA followed by Tukey's post hoc test was performed for multiple comparisons using SPSS version 21.0 software (IBM Corp.). Pearson's correlation analysis was used to analyze the relationship between LINC00858 and miR-3064-5p. P<0.05 was considered to indicate a statistically significant difference.

\section{Results}

LINC00858 is increased in bladder cancer tissues and the cancer cell lines. As revealed in Fig. 1A, RT-qPCR analysis revealed that the RNA expression level of LINC00858 was increased in the bladder cancer tissues compared with that in the paired adjacent normal tissues. Consistently with the findings in tissues, LINC00858 was revealed to be upregualted in three bladder cancer cell lines (5673, T24 and J82) compared with the normal bladder cells (Fig. 1B). All these data indicated a positive relationship between LINC00858 and bladder cancer progression.

Knockdown of LINC00858 suppresses bladder cancer cell proliferation, migration and invasion. To investigate the role of LINC00858 in bladder cancer, the specific shRNA targeting LINC00858 was used to knock down the expression level of LINC00858 both in 5637 and T24 bladder cancer cells. The transfection efficiency is presented in Fig. 2A and B. CCK-8 and colony formation assays revealed that knockdown of LINC00858 inhibited the proliferation of 5637 and T24 cells (Fig. 2C and D). It has been previously reported that the cell apoptosis of bladder cancer was induced by reactive oxygen species (ROS) (19). In the present study, it was revealed that the content of ROS was increased in the sh-LINC00858 group (Fig. 2C). Furthermore, Transwell migration and invasion assays were conducted to determine whether LINC00858 is involved in the regulation of bladder cell migration and invasion. In the present study, knockdown of LINC00858 significantly inhibited bladder cancer cell migration and invasion as evidenced by the wound healing and Transwell chamber assays (Fig. 3A and B). At the molecular level, the expression levels of migration/invasion-associated proteins, including Cox-2, MMP2 and MMP9, were markedly decreased in response to LINC00858 knockdown (Fig. 3C). These findings indicated that LIN00858 may exert an oncogenic action on the aggressiveness of bladder cells in vitro.

LINC00858 acts as a sponge for miR-3064-5p in bladder cancer cells. As revealed in Fig. 4A, bioinformatics analysis using starBase v2.0 indicated that miR-3064-5p was a 

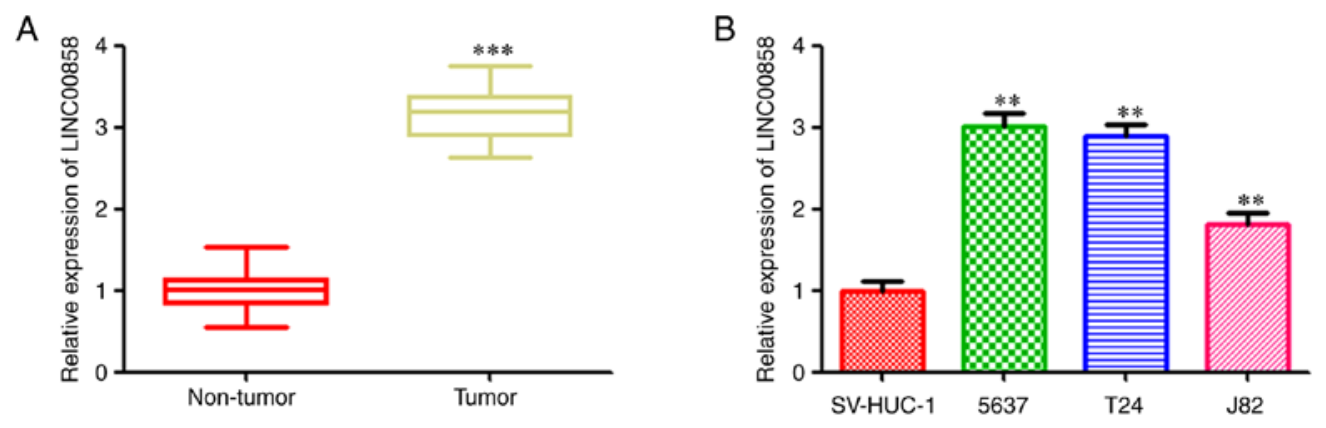

Figure 1. LINC00858 is highly expressed in bladder cancer tissues and cells. (A) RT-qPCR was performed for the examination of LINC00858 levels in healthy tissues and bladder tumor tissues. (B) RT-qPCR analysis of the RNA expression level of LINC00858 in three bladder cancer cell lines (5637, T24 and J82) and a normal human bladder epithelium cell line (SV-HUC-1). ${ }^{* *} \mathrm{P}<0.01$ and ${ }^{* * *} \mathrm{P}<0.001$ vs. the non-tumor group or SV-HUC-1 cells. All data are presented as the mean \pm SD. LINC00858, long non-coding RNA 00858; RT-qPCR, reverse transcription-quantitative polymerase chain reaction.
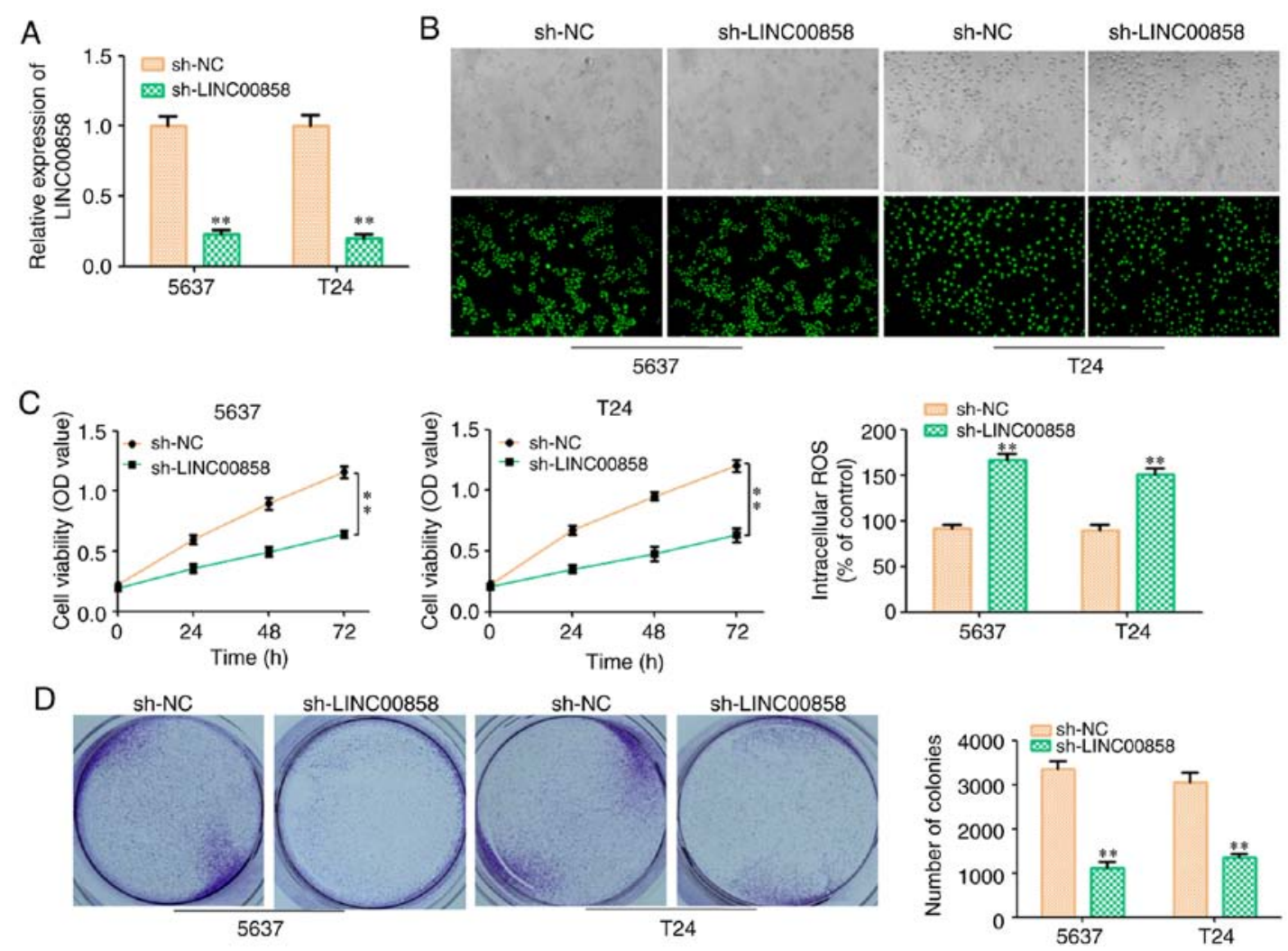

Figure 2. Knockdown of LINC00858 inhibits bladder cancer cell proliferation in vitro. 5637 and T24 cells were infected with sh-NC or sh-LINC00858. (A) RT-qPCR and (B) green fluorescence microscopy were performed to validate the transfection efficiency (scale bar, $50 \mu \mathrm{m}$ ). (C) CCK-8 assays and determination of ROS content. (D) Images of the colony formation assays and statistical calculations of the number of colonies (scale bar, $50 \mu \mathrm{m}$ ). ${ }^{* *} \mathrm{P}<0.01 \mathrm{vs}$. the sh-NC group. LINC00858, long non-coding RNA 00858; RT-qPCR, reverse transcription-quantitative polymerase chain reaction; sh-, short hairpin; $\mathrm{NC}$, negative control.

potential downstream target of LINC00858. RT-qPCR analysis revealed that miR-3064-5p was expressed at a low level in bladder cancer tissues and cells compared with normal samples and cells (Fig. 4B and C). A previous study has revealed that lncRNAs may function as ceRNAs by sponging miRNAs (20).

To validate true functional binding between LINC00858 and miR-3064-5p, luciferase reporter assays were performed. The mimics of miR-3064-5p were transfected into 5637 and T24 cells for miR-3064-5p overexpression, as confirmed by RT-qPCR (Fig. 4D). The luciferase reporter assay results revealed that miR-3064-5p mimics transfected into 5637 and
T24 cells significantly reduced the activity of the luciferase reporter, while no significant alterations were observed in the luciferase activity of mutant LINC00858 (Fig. 4E). Notably, RIP assays revealed that the expression levels of LINC00858 and miR-3064-5p in Ago2 RIP were significantly higher than those in IgG RIP, suggesting the direct binding of these two RNAs (Fig. 4F). Moreover, based on the results of the RT-qPCR assays, the relative levels of miR-3064-5p were notably increased due to silencing of LINC00858 in 5637 and T24 cells (Fig. 4G). In addition, Pearson's correlation analysis indicated that miR-3064-5p was negatively correlated with LINC00858 (Fig. 4H). 

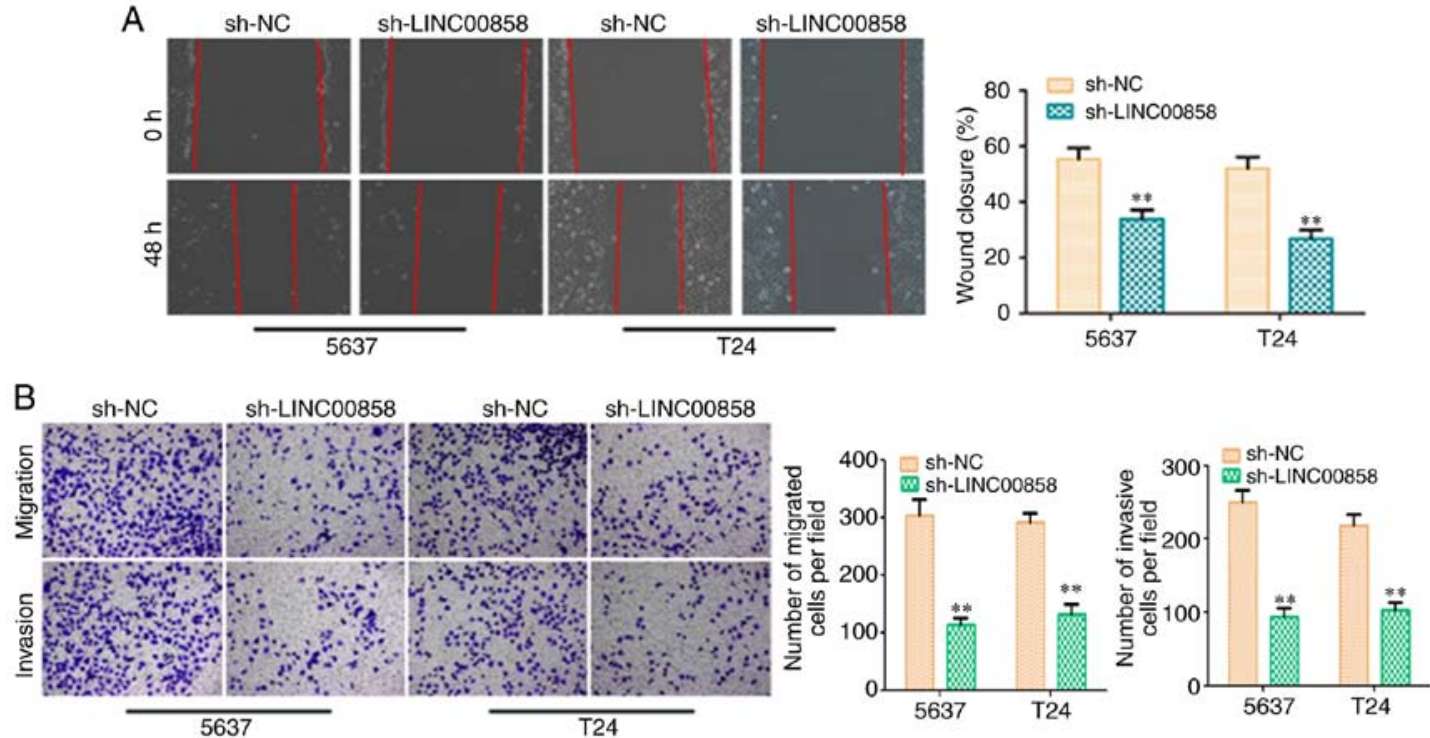

sh-

sh-
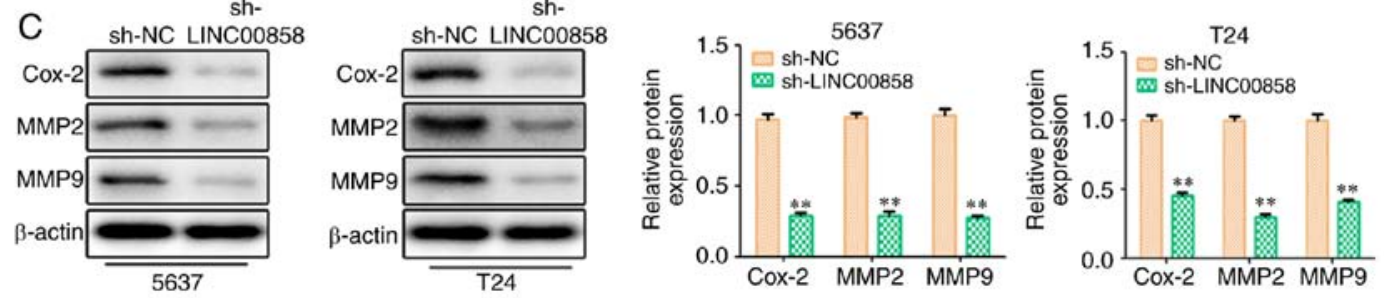

Figure 3. Knockdown of LINC00858 inhibits bladder cancer cell migration and invasion in vitro. 5637 and T24 cells were infected with sh-NC or sh-LINC00858. (A and B) Cell migration and invasion were determined by wound healing and Transwell migration and invasion assays. (C) The protein levels of Cox-2, MMP2 and MMP9 were measured by western blotting. ${ }^{* *} \mathrm{P}<0.01$ vs. the sh-NC group. LINC00858, long non-coding RNA 00858; RT-qPCR, reverse transcription-quantitative polymerase chain reaction; sh-, short hairpin; NC, negative control.

A

LINC00858-WT 5' -ugGCAUGGGAUGCCAGCCAGg-3 hsa-miR-3064-5p 3'-aaCGUGUGGUGUUGUCGGUCu-5

LINC00858-Mut $5^{\prime}$-ugCGUUGGGUUGCGUCGGUCg-3'
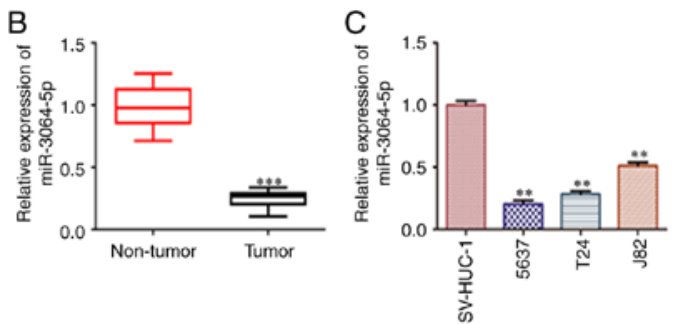
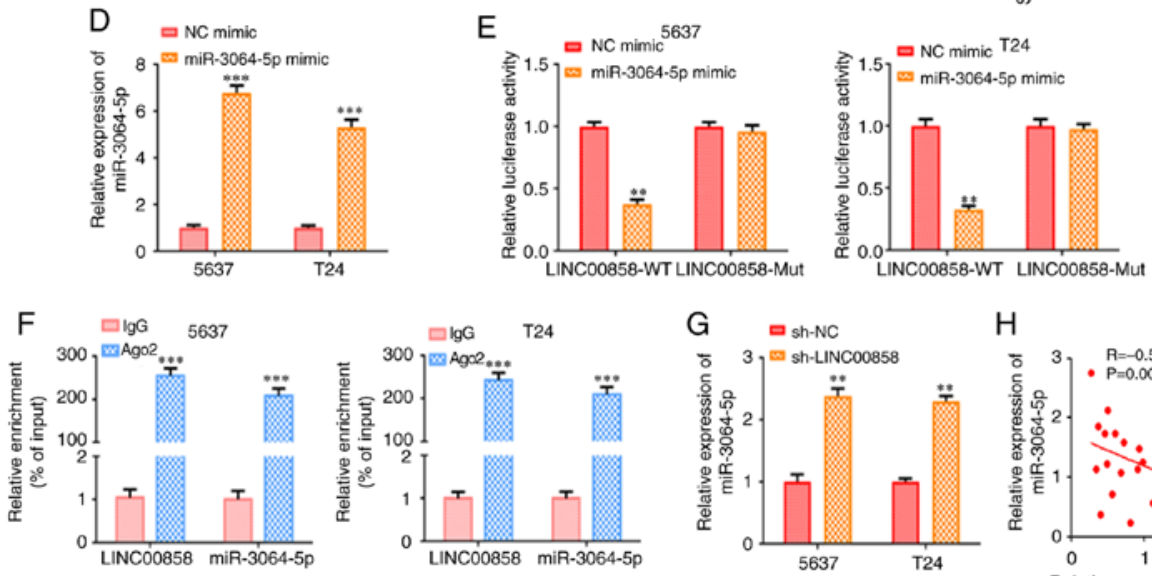

$\mathrm{H}$

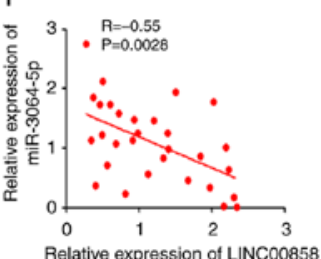

Figure 4. LINC00858 bindsto miR-3064-5p in bladder cancer cells. (A) Binding region between miR-3064-5p and LINC00858 which was predicted by starBase V2.0. (B and C) Expression levels of miR-3064-5p in bladder tumor tissues and cell lines. ${ }^{* *} \mathrm{P}<0.01$ or ${ }^{* * * *} \mathrm{P}<0.001$ vs. the non-tumor group or SV-HUC-1 cells. (D) Relative expression level of miR-3064-5p in 5637 and T24 cells transfected with miR-3064-5p mimic or NC mimic. (E) Dual luciferase reporter assays determined that LINC00858 directly targeted miR-3064-5p. (F) RIP assays revealed the enrichment of LINC00858 and miR-3064-5p. (G) Expression levels of miR-3064-5p in 5637 and T24 cells transfected with sh-NC or sh-LINC00858. (H) The expression level of LINC00858 and miR-3064-5p exhibited a negative correlation. ${ }^{* *} \mathrm{P}<0.01$ and ${ }^{* * *} \mathrm{P}<0.001$ vs. NC mimic or IgG or sh-NC. LINC00858, long non-coding RNA 00858; miR, microRNA; NC, negative control; RIP, RNA immunoprecipitation; sh-, short hairpin. 
A
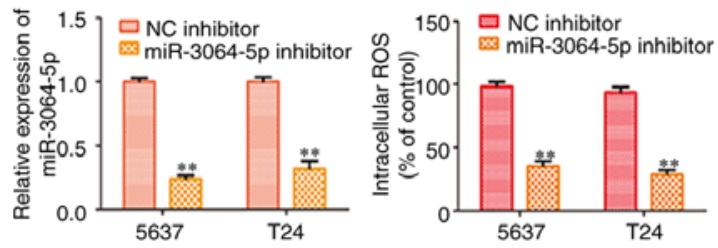

5637
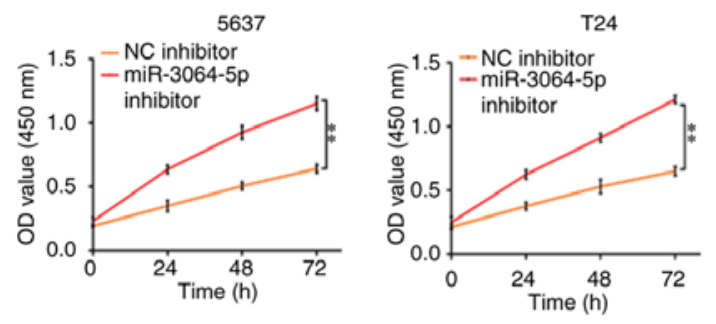

D
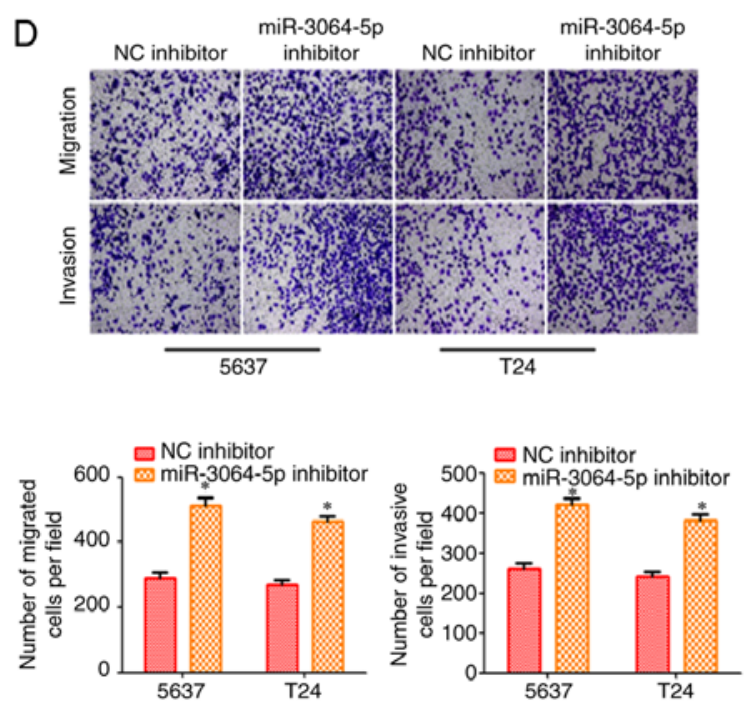
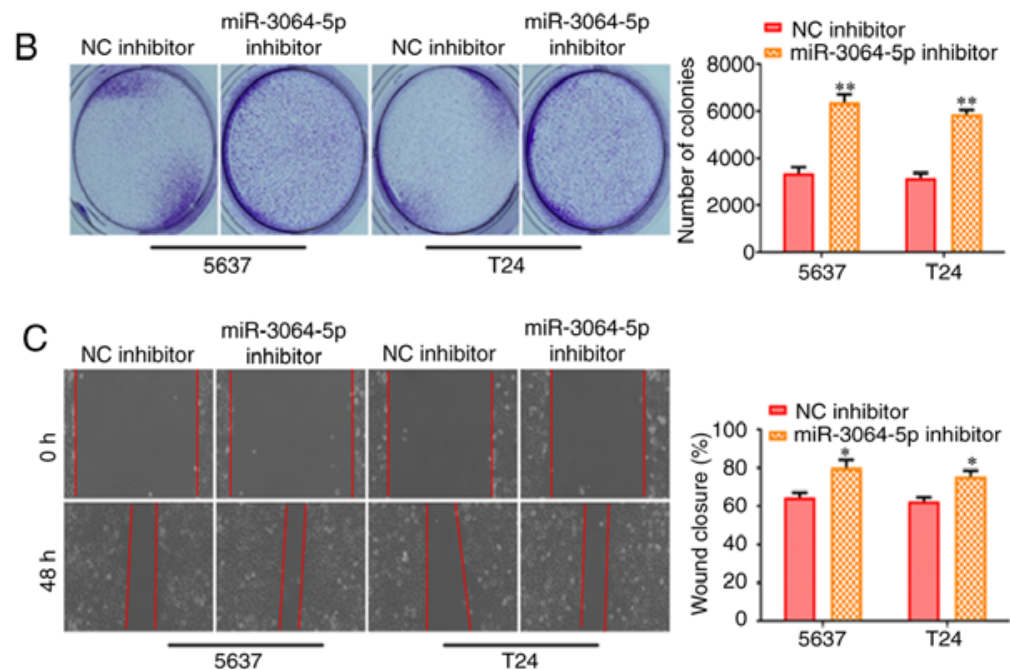

E
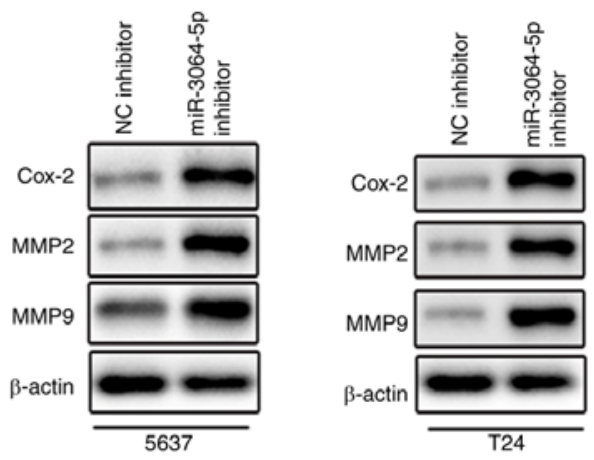

T24
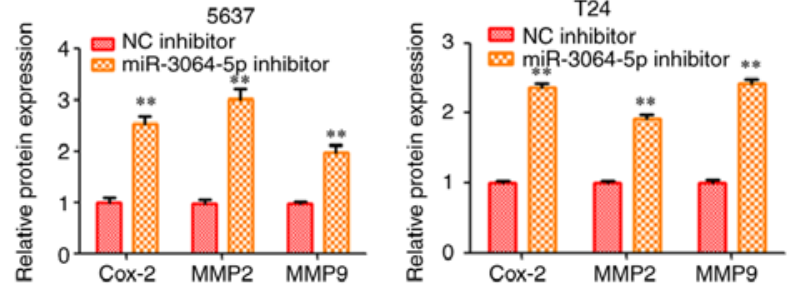

Figure 5. miR-3064-5p inhibits bladder cancer cell proliferation, migration and invasion. 5637 and T24 cells were co-transfected with sh-LINC00858 and miR-3064-5p inhibitor or NC inhibitor. (A) CCK-8 assays were performed to evaluate the cell viability, and a CellROX Green Kit was used to evaluate the content of ROS. (B) Representative photomicrographs of colony formation assay after transfection. (C and D) Wound healing and Transwell assays were performed to determine the migration and invasive capability of cells. (E) The protein levels of Cox-2, MMP2 and MMP9 were detected by western blotting. ${ }^{*} \mathrm{P}<0.05$ and ${ }^{* *} \mathrm{P}<0.01$ vs. NC inhibitor. miR, microRNA; sh-, short hairpin; LINC00858, long non-coding RNA 00858; NC, negative control; ROS, reactive oxygen species.

Inhibition of miR-3064-5p contributes to cell proliferation, migration and invasion of bladder cancer cells. The aforementioned data clearly demonstrated that LINC00858 directly interacted with miR-3064-5p. Thus, it was further investigated whether the LINC00858/miR-3064-5p axis modulated bladder cancer. 5637 and T24 cells were co-transfected with miR-3064-5p inhibitor and sh-LINC00858 (Fig. 5A). As revealed in Fig. 5A, the cell viability decreased by LINC00858 knockdown was rescued by the miR-3064-5p inhibitor, while increased ROS contents were reversed by miR-3064-5p inhibitor. miR-3064-5p inhibitor also rescued the effect of LINC00858 knockdown on bladder cancer cells as revealed inthe colony formation assay (Fig. 5B). Furthermore, the wound healing assay and the Transwell assays revealed that the migration and invasion of bladder cancer cells inhibited by sh-LINC00858 were increased by miR-3064-5p inhibitor. Cox-2, MMP2 and MMP9 protein levels that were significantly decreased by LINC00858 knockdown, were increased by miR-3064-5p inhibition. The effects of sh-LINC00858 on migration and invasion were partially attenuated by miR-3064-5p inhibitor (Fig. 5C-E).

LINC00858 modulates CTGF via competitive binding with $m i R-3064-5 p$. Using starBase v2.0, it was determined that CTGF was a downstream target gene of miR-3064-5p and the potential binding sequences are presented in Fig. 6A. Reporter gene assays in 5637 and T24 cells revealed that overexpression of miR-3064-5p led to a notable decrease in the transcriptional activity of CTGF 3'-UTR promoter constructs, while no significant changes were observed in the CTGF-Mut group (Fig. 6B). RIP assays suggested that miR-3064-5p binded to CTGF (Fig. 6C). Similar results of CTGF expression pattern at the mRNA and protein levels were observed and confirmed by RT-qPCR and western blot analyses (Fig. 6D and E). Notably, the results revealed that the expression levels of CTGF were markedly impeded by miR-3064-5p overexpression. In 

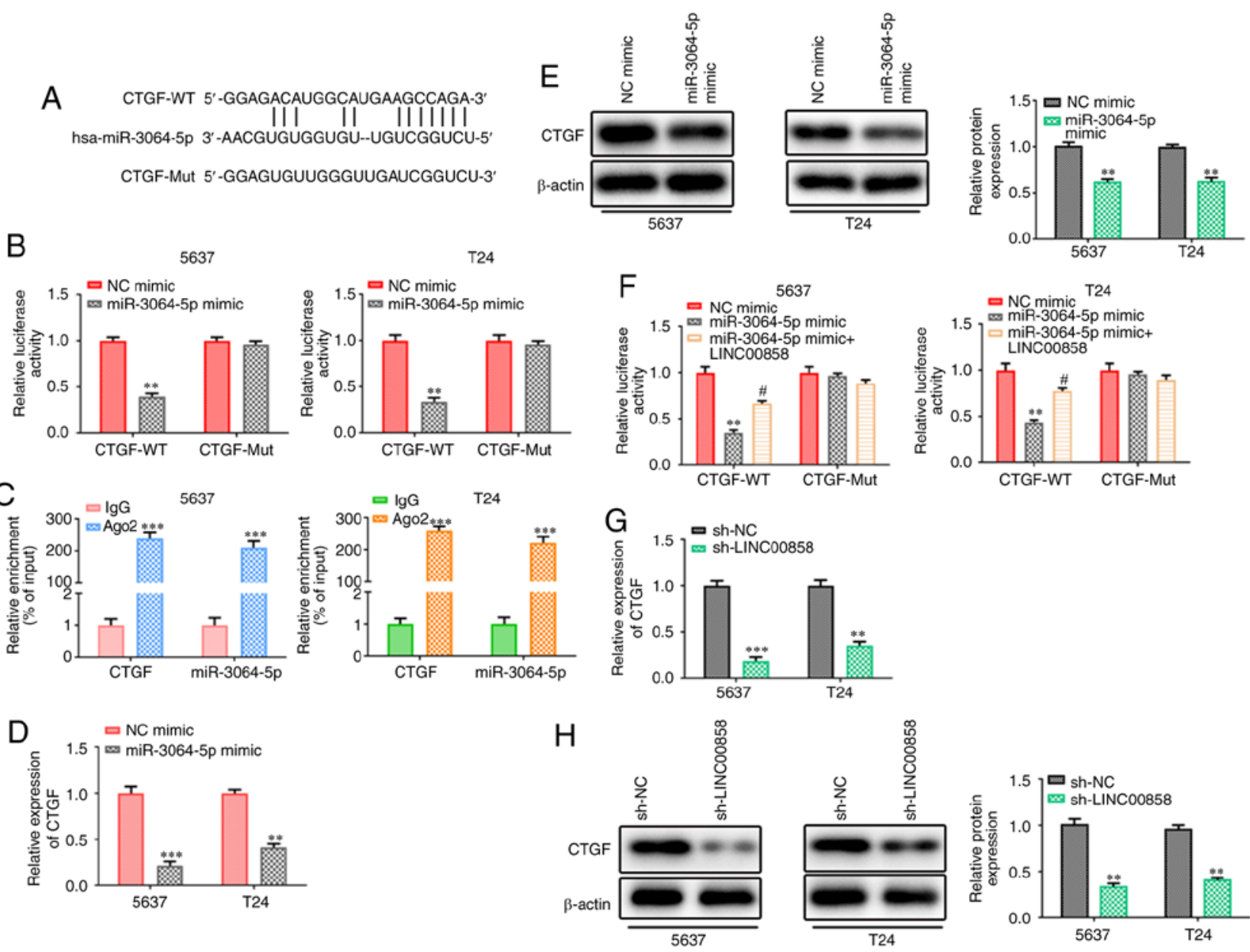

Figure 6. LINC00858 modulates CTGF via competitively binding miR-3064-5p. (A) The potential binding sequences of miR-3064-5p within the 3'-UTR of CTGF. (B) Dual luciferase activity assays and (C) RIP assays revealed that CTGF was the direct target of miR-3064-5p. ** P $<0.01$ vs. the NC mimic group for B; ${ }^{* * *} \mathrm{P}<0.001$ vs. the IgG group for $\mathrm{C}$. (D and E) The mRNA and protein levels of CTGF in 5637 and T24 cells transfected with NC mimic or miR-3064-5p mimic. ${ }^{* *} \mathrm{P}<0.01$ and ${ }^{* * *} \mathrm{P}<0.001$ vs. the NC mimic. (F) Luciferase reporter assays revealed the interaction between LINC00858, miR-3064-5p and CTGF. ${ }^{* *} \mathrm{P}<0.01$ vs. NC mimic; " $\mathrm{P}<0.05$ vs. miR-3064-5p mimic. (G and H) RT-qPCR and western blot analyses were performed to detect the expression of CTGF in 5637 and T24 cells transfected with sh-NC or sh-LINC00858. ${ }^{* *} \mathrm{P}<0.01$ and ${ }^{* * * *} \mathrm{P}<0.001$ vs. sh-NC. LINC00858, long non-coding RNA 00858; CTGF, connective tissue growth factor; miR, microRNA; RIP, RNA immunoprecipitation; NC, negative control; sh-, short hairpin.

addition, it was revealed that the miR-3064-5p-induced inhibitory effects on CTGF transcription could be partially restored by overexpression of LINC00858, indicating that LINC00858 competes with CTGF for miR-3064-5p (Fig. 6F). Finally, RT-qPCR and western blotting revealed that the expression levels of CTGF were also markedly impeded by knockdown of LINC00858 (Fig. 6G and H).

LINC00858/miR-3064-5p/CTGF axis in bladder cancer. To further study the role of LINC00858/miR-3064-5p/CTGF axis in bladder cancer, rescue assays were performed. As revealed in Fig. 7A, the transfection efficiency in 5637 was confirmed by RT-qPCR. Wound healing analysis and Transwell analysis revealed that overexpression of LINC00858 promoted cell migration and invasion, while knockdown of CTGF inhibitedcell migration and invasion (Fig. 7B-D). Moreover, western blot analysis revealed that overexpression of LINC00858 resulted in the increased expression levels of Cox-2, MMP2 and MMP9 and the effects of LINC00858 overexpression were counteracted by silencing of CTGF (Fig. 7E).

\section{Discussion}

With the advancement of RNA-Seq technologies, IncRNAs are closely associated with bladder cancer (21). Previous studies have revealed that LINC00858, a novel lncRNA, was upregulated in several cancers and functioned as a tumor promoter in colorectal cancer, non-small cell lung cancer and osteosarcoma $(8,15,22-24)$. LINC00858 promoted cell proliferation, migration and invasion by acting as a ceRNA of microRNA in the aforementioned cancers. In the present study, it was revealed that LINC00858 was aberrantly highly expressed in bladder cancer tissues and cells. Mechanism studies revealed that LINC00858 could increase the expression level of CTGF in bladder cancer cells by combining with miR-3064-5p. For the first time, to the best of our knowledge, direct evidence was provided that LINC00858 acts as an oncogene in bladder cancer and promotes the growth and metastasis of bladder cancer cells.

In addition, IncRNAs have also been revealed to act as diagnostic and prognostic biomarkersin bladder cancer (25). For instance, Shan et al have reported that 

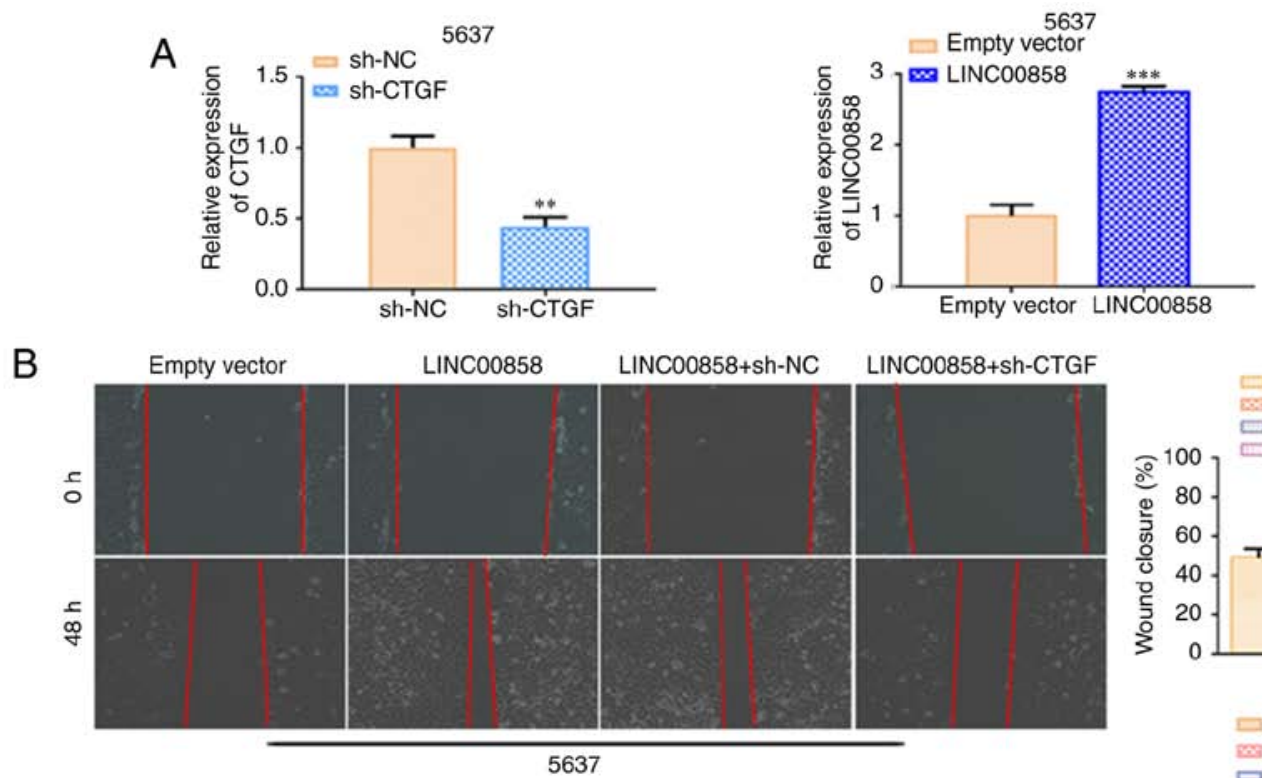

C
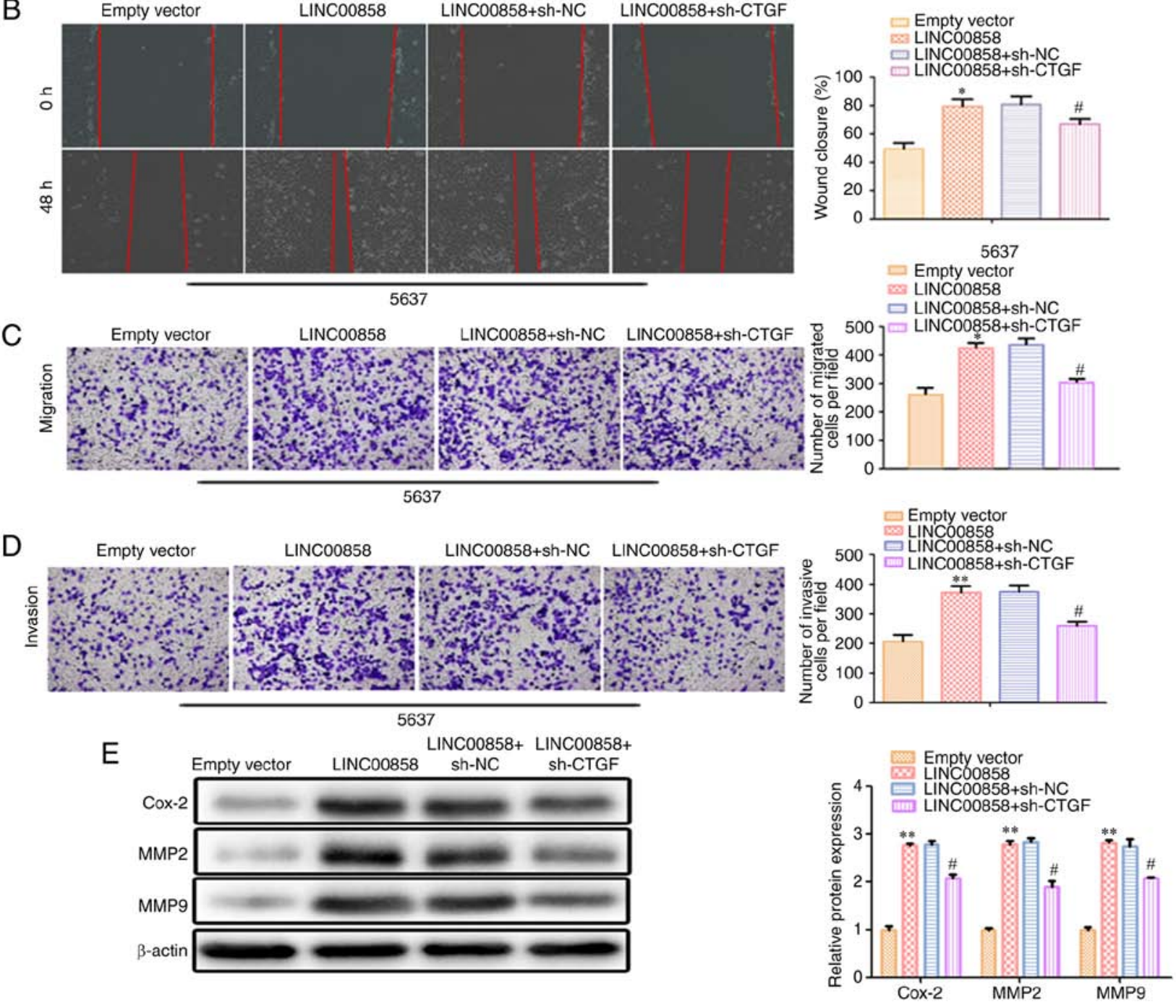

Figure 7. LINC00858 promotes bladder cancer progression by targeting the miR-3064-5p/CTGF axis. (A) The expression levels of CTGF and LINC00858 in 5637 cells transfected with sh-CTGF and LINC00858-overexpressed. ${ }^{* *} \mathrm{P}<0.01,{ }^{* * * *} \mathrm{P}<0.001$ vs. sh-NC or empty vector. (B-D) Wound healing and Transwell assays were performed to determine the migration and invasive capability of cells (scale bar, $50 \mu \mathrm{m}$ ). ${ }^{*} \mathrm{P}<0.05$ and ${ }^{* * *} \mathrm{P}<0.01$ vs. empty vector; and ${ }^{\#} \mathrm{P}<0.05$ vs. LINC00858+sh-NC. (E) The protein levels of Cox-2, MMP2 and MMP9 were detected by western blotting. ${ }^{*} \mathrm{P}<0.01$ vs. empty vector; and ${ }^{\#} \mathrm{P}<0.05$ vs. LINC00858+sh-NC. LINC00858, long non-coding RNA 00858; miR, microRNA; CTGF, connective tissue growth factor; sh-, short hairpin; NC, negative control.

lncRNA NEAT1 promoted bladder progression by regulating miR-410-mediated HMGB1 (23). Liu and Wu demonstrated that IncRNA NNT-AS1 enhanced bladder cancer cell growth by targeting the miR-1301-3p/PODXL axis and activating the Wnt pathway (24), and in 2019, Fang et al revealed that DLX6-AS1 promoted cell growth and invasiveness in bladder cancer by modulating the miR-223-HSP90B1 axis (25).

Functionally, some lncRNAs contain miRNA-binding elements and act as ceRNAs, suppressing miRNA activities. In the present study, it was revealed using a bioinformatics database that miR-3064-5p may be a target of LINC00858.
miR-3064-5p was revealed to be downregulated in cancer tissues and cells in the present study. A recent study indicated that miR-3064-5p served as a tumor suppressor in gastric cancer (26). Herein, for the first time, to the best of our knowledge, it was revealed that miR-3064-5p reversed the effects of LINC00858 on bladder cancer cells.

CTGF, encoded within chromosomal 6q23.2, has been reported to be a potential oncogene in cancer (27-29). CTGF played a crucial role for osteolytic bone metastasis both by enhancing invasiveness of tumor cells and producing RANKL for osteoclastogenesis (30). In addition, CTGF has been 
confirmed as a potential prognostic marker for medulloblastoma (31). With regard to the present research, it was revealed that CTGF was a direct target of miR-3064-5p. Moreover, it was also revealed that the expression level of CTGF was significantly decreased by knockdown of LINC00858. Knockdown of CTGF reversed the effects of LINC00858 overexpression on bladder cancer cells. Therefore, CTGF mediated the oncogenic role of LINC00858 in the development of bladder cancer.

In summary, the present study demonstrated that knockdown of LINC00858 suppressed the progression andmetastasis of bladder cancer cells in vitro. In terms of the mechanism, LINC00858 functioned as a ceRNA by regulating the expression level of CTGF by sponging miR-3064-5p.

\section{Acknowledgements}

Not applicable.

\section{Funding}

No funding was received.

\section{Availability of data and materials}

All data generated or analysed during this study are included in this published article.

\section{Authors' contributions}

JH, WMZ and XHT conceived the study and revised the manuscript. QMH and $\mathrm{CH}$ conducted all the experiments and drafted the manuscript. QW and GXW interpreted and analyzed the data. All authors reviewed, read, and approved this manuscript and agree to be accountable for all aspects of the research in ensuring that the accuracy or integrity of any part of the work are appropriately investigated and resolved.

\section{Ethics approval and consent to participate}

The present study was approved by the Ethics Committee of Jiangxi Cancer Hospital (approval no. 2019014). Written informed consent was obtained from all participants.

\section{Patient consent for publication}

Not applicable.

\section{Competing interests}

The authors declare that they have no competing interests.

\section{References}

1. Ferlay J, Soerjomataram I, Dikshit R, Eser S, Mathers C, Rebelo M, Parkin DM, Forman D and Bray F: Cancer incidence and mortality worldwide: Sources, methods and major patterns in GLOBOCAN 2012. Int J Cancer 136: E359-E386, 2015.

2. Hedegaard J, Lamy P, Nordentoft I, Algaba F, Høyer S, Ulhøi BP, Vang S, Reinert T, Hermann GG, Mogensen K, et al Comprehensive transcriptional analysis of early-stage urothelial carcinoma. Cancer Cell 30: 27-42, 2016.
3. Antoni S, Ferlay J, Soerjomataram I, Znaor A, Jemal A and Bray F: Bladder cancer incidence and mortality: A global overview and recent trends. Eur Urol 71: 96-108, 2017.

4. Renganathan A and Felley-Bosco E: Long noncoding RNAs in cancer and therapeutic potential. Adv Exp Med Biol 1008: 199-222, 2017.

5. Lenis AT, Lec PM, Chamie K and Mshs MD: Bladder cancer: A review. JAMA 324: 1980-1991, 2020.

6. Luo D, Deng B, Weng M, Luo Z and Nie X: A prognostic 4-lncRNA expression signature for lung squamous cell carcinoma. Artif Cells Nanomed Biotechnol 46: 1207-1214, 2018.

7. Yang G, Lu X and Yuan L: IncRNA: A link between RNA and cancer. Biochim Biophys Acta 1839: 1097-1109, 2014.

8. Sha QK, Chen L, Xi JZ and Song H: Long non-coding RNA LINC00858 promotes cells proliferation, migration and invasion by acting as a ceRNA of miR-22-3p in colorectal cancer. Artif Cells Nanomed Biotechnol 47: 1057-1066, 2019.

9. Shi T, Gao G and Cao Y: Long noncoding RNAs as novel biomarkers have a promising future in cancer diagnostics. Dis Markers 2016: 9085195, 2016.

10. Toiyama Y, Okugawa Y and Goel A: DNA methylation and microRNA biomarkers for noninvasive detection of gastric and colorectal cancer. Biochem Biophys Res Commun 455: 43-57, 2014.

11. Bhan A, Soleimani M and Mandal SS: Long noncoding RNA and cancer: A new paradigm. Cancer Res 77: 3965-3981, 2017.

12. Martens-Uzunova ES, Böttcher R, Croce CM, Jenster G, Visakorpi T and Calin GA: Long noncoding RNA in prostate, bladder, and kidney cancer. Eur Urol 65: 1140-1151, 2014.

13. Zhang X, Gejman R, Mahta A, Zhong Y, Rice KA, Zhou Y, Cheunsuchon P, Louis DN and Klibanski A: Maternally expressed gene 3, an imprinted noncoding RNA gene, is associated with meningioma pathogenesis and progression. Cancer Res 70: 2350-2358, 2010.

14. Wei X, Yang X, Wang B, Yang Y, Fang Z, Yi C, Shi L and Song D: lncRNA MBNL1-AS1 represses cell proliferation and enhances cell apoptosis via targeting miR-135a-5p/PHLPP2/FOXO1 axis in bladder cancer. Cancer Med 9: 724-736, 2020.

15. Xue M, Shi D, Xu G and Wang W: The long noncoding RNA linc00858 promotes progress of lung cancer through miR-3182/MMP2 axis. Artif Cells Nanomed Biotechnol 47: 2091-2097, 2019.

16. Li JH, Liu S, Zhou H, Qu LH and Yang JH: starBase v2.0: Decoding miRNA-ceRNA, miRNA-ncRNA and protein-RNA interaction networks from large-scale CLIP-Seq data. Nucleic Acids Res 42 (Database Issue): D92-D97, 2014.

17. Li J, Ma W, Zeng P, Wang J, Geng B, Yang J and Cui Q: LncTar: A tool for predicting the RNA targets of long noncoding RNAs. Brief Bioinform 16: 806-812, 2015.

18. Livak KJ and Schmittgen TD: Analysis of relative gene expression data using real-time quantitative PCR and the 2(-Delta Delta C(T)) method. Methods 25: 402-408, 2001.

19. Takeuchi H, Taoka R, Mmeje CO, Jinesh GG, Safe S and Kamat AM: CDODA-Me decreases specificity protein transcription factors and induces apoptosis in bladder cancer cells through induction of reactive oxygen species. Urol Oncol 34: 337.e11-e18, 2016.

20. Tay Y, Rinn J and Pandolfi PP: The multilayered complexity of ceRNA crosstalk and competition. Nature 505: 344-352, 2014.

21. Zhu W, Liu H, Wang X, Lu J and Yang W: Long noncoding RNAs in bladder cancer prognosis: A meta-analysis. Pathol Res Pract 215: 152429, 2019.

22. Quan J, Pan X, Zhao L, Li Z, Dai K, Yan F, Liu S, Ma H and Lai Y: lncRNA as a diagnostic and prognostic biomarker in bladder cancer: A systematic review and meta-analysis. Onco Targets Ther 11: 6415-6424, 2018.

23. Shan G, Tang T, Xia Y and Qian HJ: Long non-coding RNA NEAT1 promotes bladder progression through regulating miR-410 mediated HMGB1. Biomed Pharmacother 121: 109248, 2020.

24. Liu Y and Wu G: NNT-AS1 enhances bladder cancer cell growth by targeting miR-1301-3p/PODXL axis and activating Wnt pathway. Neurourol Urodyn 39: 547-557, 2020.

25. Fang C, Xu L, He W, Dai J and Sun F: Long noncoding RNA DLX6-AS1 promotes cell growth and invasiveness in bladder cancer via modulating the miR-223-HSP90B1 axis. Cell Cycle 18: 3288-3299, 2019.

26. Sun X, Zhang X, Zhai H, Zhang D and Ma S: A circular RNA derived from COL6A3 functions as a ceRNA in gastric cancer development. Biochem Biophys Res Commun 515: 16-23, 2019. 
27. Yang L, Hou J, Cui XH, Suo LN and Lv YW: miR-133b regulates the expression of CTGF in epithelial-mesenchymal transition of ovarian cancer. Eur Rev Med Pharmacol Sci 21: 5602-5609, 2017.

28. Alam KJ, Mo JS, Han SH, Park WC, Kim HS, Yun KJ and Chae SC: MicroRNA 375 regulates proliferation and migration of colon cancer cells by suppressing the CTGF-EGFR signaling pathway. Int J Cancer 141: 1614-1629, 2017.

29. Lun W, Wu X, Deng Q and Zhi F: miR-218 regulates epithelial-mesenchymal transition and angiogenesis in colorectal cancer via targeting CTGF. Cancer Cell Int 18: 83, 2018.
30. Kim B, Kim H, Jung S, Moon A, Noh DY, Lee ZH, Kim HJ and Kim HH: A CTGF-RUNX2-RANKL axis in breast and prostate cancer cells promotes tumor progression in bone. J Bone Miner Res 35: 155-166, 2020

31. Cruzeiro GAV, Lira RCP, de Almeida Magalhães T, Scrideli CA, Valera ET, Baumgartner M and Tone LG: CTGF expression is indicative of better survival rates in patients with medulloblastoma. Cancer Gene Ther 27: 378-382, 2020.

This work is licensed under a Creative Commons Attribution-NonCommercial-NoDerivatives 4.0 International (CC BY-NC-ND 4.0) License. 